\title{
First evidence of denticulated dentition in teleosaurid crocodylomorphs
}

\author{
Mark T. Young, Brian L. Beatty, Stephen L. Brusatte, and Lorna Steel \\ Acta Palaeontologica Polonica 60 (3), 2015: 661-671 doi:http://dx.doi.org/10.4202/app.00002.2013
}

Teleosauridae was a group of largely marine Mesozoic crocodylomorphs, typically considered as akin to "marine gavials" due to their elongate, tubular, polydont rostra that are indicative of a piscivorous diet. Here we show that these extinct crocodylomorphs were more anatomically, and perhaps ecologically, varied than previously thought. We report the first evidence of denticles in a teleosaurid tooth, revealed by scanning electron microscopic (SEM) analysis of a tooth from the holotype of "Steneosaurus" obtusidens. These denticles are cryptic, because they are microscopic, not contiguous along the carinae (instead forming short series), and are detectable only using SEM. This incipient denticle morphology is similar to that recently discovered in a closely related group of marine crocodylomorphs, the Metriorhynchidae. In particular, the denticulation morphology of "Steneosaurus " is similar to that of the geosaurin metriorhynchid Torvoneustes, indicating that these two taxa may have employed similar feeding styles and that "S." obtusidens may have been a nearshore ecological analogue to the more offshore, fast-swimming geosaurins. Previous authors have considered "S." obtusidens and Machimosaurus to be durophagous, but the discovery of denticulated teeth indicates that they had a more varied diet and feeding style, and included flesh slicing as part of their feeding toolkit. It is currently unknown how extensive denticulate carinae may be in Teleosauridae, and we hypothesise that cryptic denticles may also be present in other marine crocodylomorphs once they are subjected to SEM study.

Key words: Thalattosuchia, Teleosauridae, Steneosaurus, denticles, durophagy, functional ecology, Mesozoic, United Kingdom.

Mark T. Young [zoologika@gmail.com], School of Biological Sciences, The King's Buildings, University of Edinburgh, West Mains Road, Edinburgh, EH9 3JW, United Kingdom; and School of Ocean and Earth Sciences, National Oceanography Centre, University of Southampton, Southampton, SO14 3ZH, United Kingdom; Brian L.

Beatty [bbeatty@nyit.edu], Department of Anatomy, NYIT College of

Osteopathic Medicine, Northern Boulevard, Old Westbury, NY, 11568, USA; Stephen L. Brusatte [Stephen.Brusatte@ed.ac.uk], School of GeoSciences, Grant Institute, The King's Buildings, University of Edinburgh, West Mains Road, Edinburgh, EH9 3JW, United Kingdom; Lorna Steel [1.steel@nhm.ac.uk], Department of Earth Sciences, Natural History Museum, Cromwell Road, London, SW7 5BD, United Kingdom. 
This is an open-access article distributed under the terms of the Creative Commons Attribution License (for details please see creativecommons.org), which permits unrestricted use, distribution, and reproduction in any medium, provided the original author and source are credited.

FaF 